\title{
Applying CTA to the Design of SA-Oriented Visualizations: Heuristics and Recommendations
}

\author{
Rosa Romero $^{(\bowtie)}$, Sara Tena, David Díez, and Paloma Díaz \\ DEI Lab - Computer Science Department, \\ Universidad Carlos III de Madrid, Madrid, Spain \\ \{rmromero, stena, ddiez,pdp\}@inf.uc3m.es
}

\begin{abstract}
The effectiveness of visualizations depends on their feasibility to support the goals and tasks of the user in some particular target domain. In order to fulfill this requirement, task analysis has been characterized as a needful activity during the visualization design cycle; however, there is a lack of artefacts that guides the processing of user tasks to design visualizations. This lack of guidelines may hide the identification of such tasks that should be supported by visualization. This fact is especially relevant in operational environments in which ineffective visualization designs may hinder the acquisition of Situation Awareness (SA) and, therefore, lead to the performance of erroneous operational decisions. With the purpose of overcoming this situation, this chapter presents a set of heuristics for addressing the application of Cognitive Task Analysis (CTA) to identify visualization-supported tasks within this type of environments. The definition of such heuristics has relied on the review of the literature about SA-oriented design, visualization, and CTA, and the application of these concepts during the design of control system interfaces. The final aim is to provide a design framework that helps visualization designers to apply task analysis methods to the design of SA-oriented visualizations.
\end{abstract}

Keywords: Human-centered visualization design $\cdot$ Situation awareness $\cdot$ Goal task analysis $\cdot$ Visualization-supported tasks

\section{Introduction}

Visualization design is defined as 'the process of designing information to match the processing characteristics of human visual system' [16]. Aiming at providing effective visualizations, this process consists of a set of transformations, such as data transformations, visual mappings, or view transformations, which can be carried out through two main approaches which are different yet complementary: (1) the dataoriented approach: the accomplishment of these transformations is mainly based on data attributed characteristics such as whether an attribute is categorical, numerical, or ordinal; and (2) the human-centered approach: these transformations are not only based on data characteristics but also on the user's tasks and the environment. Over the last decade, applying the latter has led to the definition of the concept of 
human-centered visualization [9, 16], the general idea of which is that visualization should be adapted to user's needs, skills, and limitations. Based on this ground, and trying to provide some guidance to visualization designers, several authors such as Munzner [11], Wassink et al. [15], or Zhang et al. [16] have proposed models and design frameworks that describe the structure of tasks, users, and functions. Although these models have clear differences among them, they concur in characterizing task analysis as a required process for effective human-centered visualization designs. Unfortunately, under the umbrella of task analysis, and the definition of the task performed by the user throughout an activity, there are numerous methods, approaches, and techniques to consider; most of which have not been conceived to fulfil the study of specific domains and systems. Furthermore, since the main challenge in designing visualizations is not only the gathering of the tasks carried out by the user but also the suitable characterization of those tasks, the application of task analysis techniques to the design of visualization is not trivial; applications that have so far mainly relied on the experience of visualization designers. The lack of context-oriented guidelines may cause the highlighting of irrelevant tasks or the collection of inadequate information for a successful visualization design.

The design of unfitting visualization is especially problematic in operational environments in which visualizations support the performance of critical tasks. Operational environments refer to working situations in which an operator controls the functioning of either a critical process or an essential infrastructure. During their activity, operators must be aware of all the incidents, actions, and situations related to the operating situation, monitoring and controlling the state of either the process or the infrastructure. In these environments, the acquisition of Situation Awareness (SA) is a key factor to perform the operation of these critical process and infrastructures [4]. In fact, the analyses of recent problems in a variety of operational environments show the relevance of SA as a major factor in failures propagation [8].

Acquiring SA refers to knowing what is happening around the operation and what that information means at this moment and in the future. SA is therefore a cognitive process directly dependent on the context [6]. Thus, the design of SA-oriented visualizations requires the identification of such context goals that must be achieved by operators as well as the task performed to accomplish such goals and the information required to carry out those tasks. Figure 1 illustrates the detailed scope of SA-oriented visualizations. According to the SA-oriented visualization approach, two different but complementary disciplines guides the design of visualization: Visualization and Human-Computer Interaction (HCI). In this way, Cognitive Engineering methods such as task analysis or cognitive task analysis- allow designers to identify those tasks that must be carried out by users to achieve specific operational goals. Once these goals, and their corresponding tasks, had been identified, designers could apply different theoretical and methodological visualization foundations to create such information visualizations that assist the achievement of SA. These foundations will be supplemented by a set of SA-oriented design principles related to the design of operational user interfaces. Consequently, at this design approach the key design element will not be the operational information to represent, but the operational goals to achieve by the operators. 


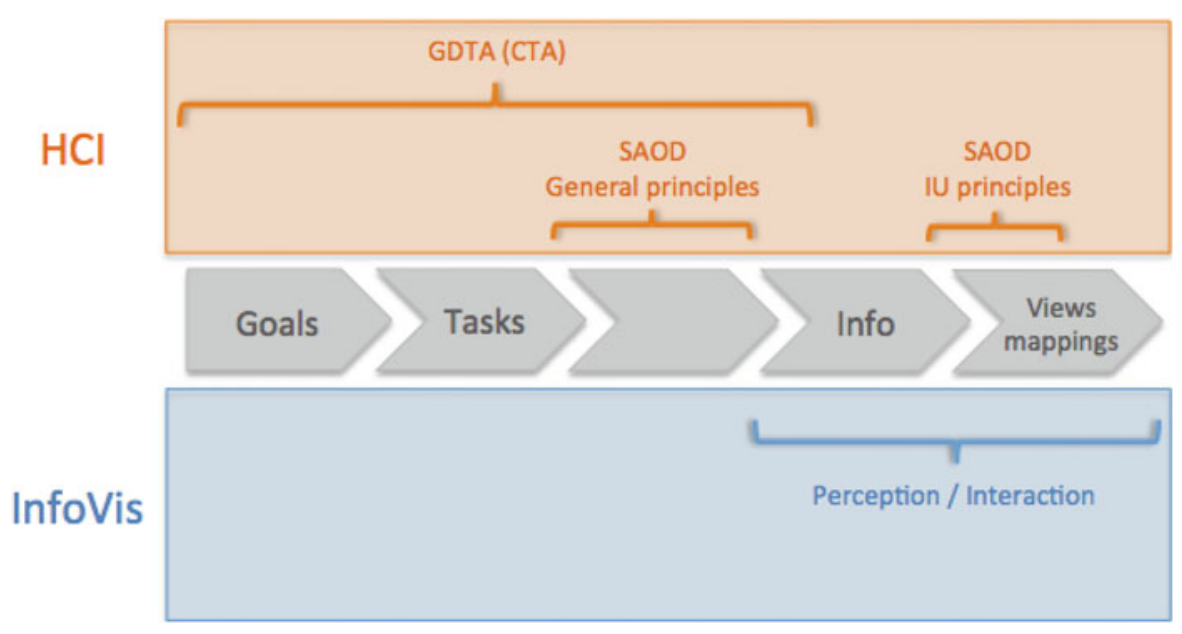

Fig. 1. The scope of SA-oriented visualizations. The design of operational visualization is not guided by the information to represent but to the operational goals to achieve.

This chapter presents a set of heuristics to guide visualization designers during the Goal-Directed Task Analysis (GDTA) of operational environments. GDTA is a CTA method specially oriented to elicit SA requirements within operational environments. The purpose of these heuristics is to reduce the relevance of the visualization designer's experience by systematizing the performance of GDTA within this context. The rest of the chapter is organized as follows. The rationale for defining these heuristics is described in the next section. Section 3 is focused on presenting the theoretical foundations that ground these heuristics. Section 4 establishes these heuristics grouped by three purposes of the task analysis process: the elicitation of visualization-supported tasks, the identification of information requirement to the task at hand, and the characterization of such information requirements. Finally conclusions and recommendations for further work are drawn in the last section.

\section{Designing for Achieving Situation Awareness}

Nowadays, SA is one of the most relevant concepts of cognitive engineering, one of the foundations for decision-making and performance, and a relevant factor studied in wide-ranging fields. Endsley formally defined SA as 'the perception of the elements in the environment within a volume of time and space, the comprehension of their meaning, and a projection of their status in the near future' [6]. Much of the operator's efforts in operational environments are devoted to developing SA and keeping it up to date [7]. As a consequence, designing artefacts for supporting this process is a challenging activity, which has been mostly carried out through the SA-oriented design process. This process model, as shown in Fig. 2, is divided into three basic stages: analysis, design, and measurement. According to the scope of this chapter, this section characterizes the first stage, highlighting the deficiencies of the analysis phase from the viewpoint of visualization design. 


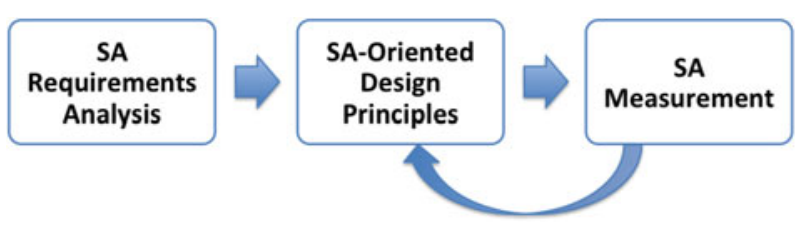

Fig. 2. SA-oriented design process [4]

The SA-oriented design (SAOD) strategy is based on the idea that SA is fundamental in driving human decision-making in complex, dynamic environments [7]. Since decision-making is shaped by goals and tasks, the SA requirement analysis stage therefore should be addressed by goals and tasks. SA requirements focus on the dynamic information requirement relevant in a particular domain rather than the static knowledge the operator must possess, such as system knowledge or rules, and procedures [6]. These SA requirements are delineated through GDTA. GDTA is a CTA method oriented to seek and document what information operators need to perform their job and how each operator integrates or combines the information to address a particular decision [4]. Conforming to that, this method involves knowledge elicitation sessions with experts aiming at providing an analysis of the information required for each level of SA linked to each goal and task. The steps involved in GDTA are presented below:

1. Review of the domain: basic information about the domain of application is crucial for designing systems.

2. Initial interviews: the key operators that are playing significant roles should be chosen for applying the GDTA method.

3. Development of a goal hierarchy: identification of the major goals and associated tasks should be extracted from the interviews.

4. Identification of decisions and SA requirements: experts should be enquired about the tasks that are necessary for accomplishing the main goal. The sub goals would serve to set the direction for clarifying the primary decision needed for each subgoal and the information needs to accomplish those sub-goals.

5. Reviewing the goal hierarchy: additional interviews to experts for reviewing the goal hierarchy are recommended in order to consolidate the information extracted.

6. Review of the GDTA: a final review of the final GDTA structure obtained from the application of the method.

Unfortunately, the application of this analysis depicts when it must be carried out, but does not support the how, which must rely on designers' experience. Moreover, since not every SA requirement is relevant from the viewpoint of visualization, designers must be able to distinguish among them for a successful visualization design. An appropriate application of the GDTA method helps designers determine ways to present information to operators in a better way in order to support SA, and consequently, in decision making and performance [6]. 


\section{SA-Oriented Visualizations: Theoretical Foundations}

Although the primacy of designer's experience is to carry out GDTA techniques, there exists a basis of knowledge related to both SAOD and visualization design that can be employed by designers to systematize the application of GDTA methods. This basis of knowledge may be considered as a grounded theory for the definition of specific design guidelines.

\subsection{Principles for Situation Awareness-Oriented Design}

The way in which information is presented to the operator through the interface greatly influences SA $[6,13]$. The most applied principles for creating SA-oriented designs are the fifty design principles proposed by Endsley [4, 5]. These principles are based on a model of human cognition involving dynamic switching between goaldriven and data-driven processing and feature support for limited operator resources. However, they underpin not only SA design interface issues but also how to design automated systems, dealing with complexity or uncertainty. For this reason, the set of principles to consider for designing effective visualizations should be reduced to those focused on the interface design.

- Goal-oriented information displays. Goal-oriented information displays should be provided, organized so that the information needed for a particular goal is colocated and directly answers the major decisions associated with the goal.

- Direct presentation of higher-level SA needs (comprehension and projection), rather than supplying only low-level data that operators must integrate and interpret manually. As attention and working memory are limited, the degree to which displays provide information that is processed and integrated in terms of comprehension and projection will positively impact SA.

- Support for global SA. Providing an overview of the situation across the operator's goals at all times and enabling efficient and timely goal switching and projection.

- Critical cues related to key features of schemata need to be determined and made salient in the interface design. In particular those cues that will indicate the presence of prototypical situations will be of prime importance and will facilitate goal switching in critical conditions.

- Support for parallel processing. Multi-modal displays should be provided in data rich environments.

- Use information filtering carefully. Extraneous information not related to SA needs should be removed (while carefully ensuring that such information is not needed for broader SA needs).

\subsection{Visualization Design Principles}

A first step in developing effective visualizations is to understand how they enable perception and cognition. The achievement of this purpose encompasses the application of the following set of widely accepted visualization design principles $[2,10,12,14]$. 
- Appropriateness principle. Visualizations should provide neither more nor less information than that needed for solving the problem.

- Naturalness principle. Experiential cognition is most effective when the properties of the visual representation most closely match the information being represented. This principle supports the idea that new visual metaphors are only useful for representing information when they match the user's cognitive model of the information. Purely artificial visual metaphors can actually hinder understanding.

- Matching principle. Representations of information are most effective when they match the task to be performed by the user. Effective visual representations should present affordances suggestive of the appropriate action.

- Principle of congruence. The structure and content of visualization should correspond to the structure and content of the desired mental representation. In other words, the visual representation should represent the important concepts in the domain of interest.

- Principle of apprehension. The structure and content of visualization should be readily and accurately perceived and comprehended.

- Principle of expressiveness. The visualization contains all the facts in the data set and only the facts.

- Principle of effectiveness. The visualization conveys the information in an effective way.

\section{SA-Oriented Visualizations: A Prescriptive Analysis Artefact}

GDTA has emerged as an essential approach to study operational environments and address the design of control systems. However, as aforementioned, there is a lack of prescriptive analysis artefacts that systematize the SA requirements analysis for designing SA-oriented visualizations. One solution to this shortcoming will be the collection of heuristics that guides the application of GDTA for designing effective visualization for operational environments. The following subsections explain the rationale of the solution as well as the defined heuristic.

\subsection{The Rationale of the Solution}

A heuristic refers to a generalizable abstraction based on the design experience. A heuristic provides an educated design rule, a simplification that can be used to address the definition of a fitting solution. In this way, heuristics are less consistent and generalizable than guidelines or principles, but they are a useful mechanism to scope a solution in emerging domains. Moreover, heuristics have proved as an appropriate mechanism to guide creative-oriented processes. The definition of rules based on previous experience and well-known practices allows reusing procedural knowledge, reducing the relevance of the design experience. 
The definition of the heuristics is based on the review of the literature about SAoriented design, visualization, and GDTA as described in Sects. 2 and 3, as well as the application of these concepts during the design of control system interfaces. In particular, the heuristics have been defined following a systematic approach:

- After reviewing the literature on SA-oriented design, principles related to the design of operational systems were collected $[4,5]$. As mentioned before in Sect. 3.1, these principles were analysed and filtered, considering only those principles related to the design of the user interface. Similarly, the most widely accepted visualization design principles (see Sect. 3.2) were selected.

- Secondly, and taking into account the GDTA method, the main goals of the design process of control systems were highlighted. For each of these goals, the literature on visualization design was considered to identify a set of experienced-based design rules. For instance, related to the elicitation of supported tasks, taxonomy of highlevel visualization task [1] was reviewed, identifying such tasks about the review of great pieces of information and the recognition of unexpected situations. Based on these references, the first draft of the heuristic was created.

- Finally, the description of the heuristic was carried out. These descriptions were based on the principles selected on the first stage of the process. In case of not obtaining a coherent and consistent description underpinned by the principles, the heuristic was withdrawn.

\subsection{Heuristics for Cognitive Task Analysis}

The heuristics defined cover three purposes of the task analysis process: (1) the elicitation of visualization-supported tasks, which is devoted to identify those relevant tasks to the visualization design; (2) the identification of information requirement to the task at hand, which is focused on gathering relevant information from users to the task at hand; and (3) the characterization of such information requirements, which is related to characterize the information requirement attributes. Following are the heuristics presented according to the purpose to which they are referred. With the goal of illustrating them, they will provide an example in the power transmission domain for each heuristic. The goal of power transmission operators is to get an efficient, safe, and sustainable power transmission. With such an aim, operators must manage on real-time large volumes of multidimensional data streams, for which extensive visualization are used. A summary of theseheuristics is provided in Table 1.

\section{The Elicitation of Visualization-Supported Tasks}

This first group of heuristics is intended to characterize those operators' tasks whose performance may be enhanced by the use of visualizations. Accordingly, and based on the taxonomy of high-level visualization tasks previously mentioned, a set of highlevel abstract goals shared by those visualization-supported tasks has been compiled. This set of high-level abstract goals is classified into three categories: (1) The management of data spread across multiple sources. SA within operational environments involves being aware of what is happening across many aspects of the environment; (2) The monitoring of a large number of potential events. Achieving good SA in operational environments is based on understanding what the data and cues perceived 
Table 1. Heuristics summary

\section{The elicitation of visualization-supported tasks}

H1 Overview tasks. Tasks that involve the acquisition of a general qualitative awareness of one aspect of some data, preferably in a very short period of time

H2 Attention sharing tasks. Tasks that involve multiple pieces of information that must be processes simultaneously

H3 Pattern recognition tasks. Tasks that involve the extraction of patterns from data

H4 Cause and effect tasks. Tasks that involve an understanding of what assumptions have gone into creating data and thus affected the outcomes inferred

\section{The identification of information requirements to the task at hand}

H5 Significant information. Information sources that are relevant to the operator's task at hand

H6 Priority of the information. The importance assigned to such information according to the operators' task at hand

H7 Relationship between pieces of information. Potential relationships among the pieces of information

H8 Modes of obtaining information. How the operators obtain the required information to the task at hand

\section{The modeling data stage}

H9 Data attributed characteristics. Data attributed must be defined to properly perform visual mappings

H10 Structural relationships among data. Structural relationships existing among data

H11 Volume of data handling. The amount of data that must be handled to the task at hand

mean in relation to relevant goals and objectives; and (3) The recognizing of patterns in information. To achieve SA, the operators try to establish correlations among data in order to comprehend what is its significance. Relying on these categories, the heuristics defined are:

- H1. Overview tasks. It is necessary to characterize those tasks that involve the acquisition of a general qualitative awareness of one aspect of some data, preferably acquired in a very short period of time.

In the electrical grid domain, the operator should be able to comprehend the current condition of the grid infrastructure at any given point in time. Thus, an overview of the various attributes of alarms is needed. An example of this overview would be the display of the distribution of the number of active alarms on a specific grid area or the typology of alarms that is contributing most to the failures.

- H2. Attention sharing tasks. It is necessary to identify those tasks that involve multiple pieces of information that must be processed simultaneously.

In the electrical grid domain, a grid operator must concurrently perform planned operations over the grid infrastructure and monitor the grid status in order to both detect and register potential incidents. Hence, managing potential incidents is a task of interest from the viewpoint of the design of SA-oriented visualizations. 
- H3. Pattern recognition tasks. It is necessary to characterize those tasks that involve the extraction of patterns from data. These tasks are related to the requirement of both integrating and prioritizing the data to achieving comprehension of the current situation.

An abnormal situation can lead to an avalanche of alarms, which grid operators need to interpret in order to identify both its origin and seriousness. Therefore, handling alarms may take advantage of the capabilities provided by SA-oriented visualizations.

- H4. Cause and effect tasks. It is necessary to characterize those tasks that involve an understanding of what assumptions have gone into creating data and thus affects the outcomes inferred.

During an avalanche of alarms, operators must project the grid infrastructure status and the likely effects of their own actions. These two tasks are potential candidates from the viewpoint of the SA-oriented visualization design.

\section{The Identification of Information Requirements to the Task at Hand}

Since the last purpose of visualization is to display information that matches the processing characteristics of the human visual system, performing GDTA for designing visualizations should involve not only the identification of the cognitive tasks related to operators' goals but also the information required to carry out these tasks. Accordingly, the following heuristics are defined in order to guide the requirement gathering about relevant aspects of this information from the viewpoint of visualization:

- H5. Significance information. It is necessary to ask for only those information sources that are relevant to operators' task at hand. Additional information may be distracting and makes the task more difficult.

While an operator must be aware of both planned operations over the grid and the status of different electrical assets for monitoring tasks, the operator does not need to know the number of customers of the utility. In this way, questions about which pieces of information are more relevant for carrying out monitoring tasks must be formulated.

- H6. Priority of the information. A different priority level exists in the information. Hence, it is necessary to ask for the importance assigned to such information according to the operators' task at hand.

Alarms are the essential mechanisms used to interpret and correct abnormal situations over the grid instead of monitoring power values. Thus, questions about which information has more priority or relevance to manage abnormal situations must be conducted. 
- H7. Relationship between pieces of information. It is necessary to characterize the potential relationships among the pieces of information.

The information provided by the field personnel is essential to carry out safe planned operations over the grid. Such information must be provided in relation with infrastructure devices. Accordingly, questions about any relationship existing between the information provided by external actors and other pieces of information managed by primary actors must be formulated.

- H8. Modes of obtaining information. It is necessary to characterize how the operators obtain the required information to the task at hand. Operators may need to explore the information space in order to obtain the needed pieces of information.

Operators may navigate across several static lists of alarms in order to gain understanding about the status of a fragment of the grid affected by an incident. Thus, questions focused on how the operators retrieve the required information to perform their tasks must be conducted.

\section{The Characterization of Information Requirements}

The purpose of this last group of heuristics is to guide the definition and detail of the tasks previously identified. This effort leads to the characterization of their information requirements. Accordingly, this characterization will serve as the input for designing SA-oriented visualizations:

- H9. Data attributed characteristics. It is necessary to characterize the data attributed characteristics in order to perform visual mappings properly.

Monitoring the grid status is mainly based on the information supplied by SCADA systems. Therefore, the design of visualization requires the knowledge of the number of dimensions of this data and the type of values. In this case, SCADA systems produce multidimensional and alphanumerical data.

- H10. Structural relationships among data. It is necessary to characterize the structural relationships existing among data.

Based on structural relationships among alarms such as time or topological relationships among electrical assets, operators may be capable of understanding the seriousness of the problem. Accordingly, it is necessary to make the type of existing relationships among alarms explicit in order to design effective visualizations.

- H11. Volume of data handling. It is necessary to characterize the amount of data that must be handled to the task at hand in order to determine the visual scalability required. 
During abnormal situations, the number of alarms registered doubles, which may hinder the achievement of SA by operators. Thus, it is necessary to identify the number of alarms that operators must handle to diagnose a problem. This number of alarms will be dependent on the operational situation.

\section{Conclusions}

Embedding knowledge into digital representations is a well-known mechanism for increasing the cognition of the operator and a manner of easing the performance of operating tasks. In that way, most operation centres are comprised of a set of technological devices that deploy digital representations about the system to operate. It means that operators are mainly in contact with the control system they operate via the information provided by these digital representations. In particular, the use of visualizations has been highlighted as an essential artefact to support both the understanding of the operators about the system and the performance of appropriate actions. Similarly, SA is considered as the most important factor that determines the performance of the operators. SA involves being aware of what is happening in the system to operate and how these facts could impact immediately and in the near future. Unfortunately, in spite of their mutual arrangement and relation, there is a lack of design artefacts that lead the definition of visualization specially conceived to provide SA. SA-oriented design methodologies are focused on identifying such labours that must be fulfilled by the operator, leaving aside which information should be managed to perform those labours or which of them could be better accomplished by the use of digital representations. Besides, the criticality of the operational environments hinders the application of the usual interactive design techniques such as prototyping. Thus, the definition of prescriptive artefacts comes up as an essential requirement to address the design of SA-oriented visualizations.

One of the most important activities in design, in general, and in SA-oriented design, in particular, is the inquiry process. The definition of rules based on previous experience and well-known practices allows reusing procedural knowledge, reducing the relevance of the design experience. The review of literature related to SA-oriented design and GDTA, and the application of both concepts during the design of control system interfaces has allowed us to identify a set of tasks that may take full advantage of visualization power. In particular, tasks such as overview tasks, attention sharing tasks, pattern recognition tasks, and cause and effect tasks have been identified as the most significant visualization-supported tasks to provide SA. Additionally, a set of guides about what kind of information and which dimensions should be collected to design appropriate visualizations has been defined. Dimensions such as the significance of the data, its priority, relationship, and modes of obtaining information are necessary to ensure that extra features not required by the task at hand are included by these visualizations. 
Operational environments, as critical working settings, can be regarded as a very procedural environment, in which the operators perform their tasks in keeping with well-known procedures and protocols. Accordingly, the specification in advance of the operational goals is competently achievable. Nonetheless, there are unexpected and unusual situations, situations defined as crisis situations, which cannot be completely specified in advance. Consequently, our approach can be considered as an appropriate solution to define such visualizations that assist regular operational conditions. Even so, since during crisis situations the operation is basically based on the ability, experience, and training of the operators, moving the digital representation to the background, this limitation barely restricts the usefulness of our solution.

Further work will lead to the application of these heuristics to other different operational domains for refinement or refutation. Subsequently, these heuristics will be extended to address the application of SA-oriented design principles. Such principles provide a blueprint for considering SA aspects into designs but its proper application is also mainly dependent on the designer's experience. As a second step, the limitation of our approach to crisis situations will be faced. In particular, other cognitive engineering frameworks, such as the Cognitive Work Analysis (CW) [14] or the Work-Centered Design (WCD) [3], should be analysed to identify the keys of building systems that facilitate SA in unexpected situations. The final aim of our work is to define a design framework that addresses the human-centered visualization paradigm in operational environments.

Acknowledgments. This work has been supported by the ENERGOS Project, funded by the Centre for Industrial Technological Development (CDTI) of the Ministry for Science and Innovation of Spain.

\section{References}

1. Amar, R., Stasko, J.: A knowledge task-based framework for the design and evaluation of information visualizations. In: IEEE Symposium on Information Visualization (Infovis), pp. 143-149 (2004)

2. Card, S., Mackinlay, J., Shneiderman, B.: Readings in Information Visualization. Morgan Kaufmann, San Francisco (1999)

3. Eggleston, R.G.: Work-centered design: a cognitive engineering approach to system design. In: Proceedings of the Human Factors and Ergonomics Society, pp. 265-269 (2003)

4. Endsley, M.R.: Design and evaluation for situation awareness enhancement. In: Proceedings of Human Factors Society 32nd Annual Meeting, Santa Monica (1988)

5. Endsley, M.R.: Toward a theory of situation awareness in dynamic systems. Hum. Factors 37(1), 32-64 (1995)

6. Endsley, M.R., Bolté, B., Jones, D.G.: Designing for Situation Awareness. Taylor \& Francis, New York (2003)

7. Endsley, M.R., Bolstad, C.A., Jones, D.G., Riley, J.M.: Situation awareness oriented design: from user's cognitive requirements to creating effective supporting technologies. Hum. Factors Ergon. Soc. Annu. Meet. 47, 268-272 (2003) 
8. Greitzer, F.L., Schur, A., Paget, M., Guttromson, R.T.: A sensemaking perspective on situation awareness in power grid operations. IEEE Power and Energy Society General Meeting Conversion and Delivery of Electrical Energy in the 21 st Century. IEEE, Pittsburgh (2008)

9. Kerren, A., Ebert, A., Meyer, J.: Human-Centered Visualization Environments. Springer, Berlin (2006)

10. Mackinlay, J.: Automating the design of graphical presentations of relational information. ACM Trans. Graph. 5(2), 110-141 (1986)

11. Munzner, T.: A nested model for visualization design and validation. IEEE Trans. Vis. Comput. Graph. 15(6), 921-928 (2009)

12. Norman, D.: Things That Make Us Smart: Defending Human Attributes in the Age of the Machine. Perseus Books, New York (1993)

13. Rothenberg, D.: Alarm Management for Process Control. Monumentum Press, New Jersey (2009)

14. Tversky, B., Morrison, J.B., Betrancourt, M.: Animation: can it facilitate? Int. J. Hum.Comput. Stud. 57(4), 247-262 (2002)

15. Wassink, I., Kulyk, O., van Dijk, E.M.A.G., van Veer, G.C, van der Vet, P.E.: Applying a user-centred approach to interactive visualization design. Trends in Interactive Visualization. Advanced Information and Knowledge Processing. Springer, London (2008)

16. Zhang, J., Johnson, K.A., Malin, J.T.: Human-centered information visualization. In: International Workshop on Dynamic Visualization and Learning, Tubigen (2002) 